\title{
NON-HYPERELLIPTIC RIEMANN SURFACES
}

\author{
RICHARD S. HAMILTON
}

1. Let $W$ be a Riemann surface of genus $g \geq 2$. Abel's Theorem gives an analytic embedding, with respect to an arbitrary base point, of $W$ as a submanifold of its Jacobi variety $J(W)$ (see Gunning [1, p. 161]). Denote by $W_{r}$ the linear equivalence classes in $J(W)$ of divisors consisting of $r$ points in $W$. Then $W=W_{1}$ and $W_{r}$ is a subvariety of dimension $r$ for $0 \leq r \leq g$. More generally, let $W_{r}^{a}$ denote the set of all linear equivalence classes of divisors of degree $r$ in $J(W)$, which admit at least a linearly independent meromorphic function, or equivalently, the set of all line bundles on $W$ of Chern class $r$ which admit at least a linearly independent analytic section. Then $W_{r}=W_{r}^{1}$, and $W_{r}^{a}$ is a subvariety of $J(W)$. Alan Mayer [4] showed that $\operatorname{dim} W_{r}^{a} \leq r$ $-2 a+2$ (provided $1 \leq r \leq g-1, a \geq 2$, and $r-2 a+2 \geq-1$ ) and that the maximum is in fact attained whenever $W$ is hyperelliptic. He then conjectured that the converse was true, and this is our main result.

Theorem 1. If $W$ is not hyperelliptic, then $\operatorname{dim} W_{r}^{a} \leq r-2 a+1$ (provided $1 \leq r \leq g-1, a \geq 2$, and $r-2 a+1 \geq-1)$.

Thus surfaces which are not hyperelliptic have fewer "special" divisors than those which are.

2. Before proceeding to the proof of Theorem 1, it may be of interest to see how two classical theorems on non-hyperelliptic surfaces may be deduced from this result.

Clifford's Theorem. If $W$ is not hyperelliptic, then no translate of $W_{r}$ is contained in $-W_{r}$, for $1 \leq r \leq \mathrm{g}-2$.

Proof. The set of all elements $x$ such that the translate of $-W_{r}$ by $x$ is contained in $W_{r}$ is the set $W_{r} \ominus-W_{r}=W_{2 r}^{r+1}$ (see $\S 3$ ). Hence the theorem is equivalent to the assertion that if $W$ is not hyperelliptic then $W_{2 r}^{r+1}$ is empty. If $2 r \leq g-1$, Theorem 1 states that $\operatorname{dim} W_{2 r}^{r+1} \leq-1$. If $2 r \geq g-1$, let $k$ be the divisor class in $J(W)$ of an abelian differential. Then $k-W_{2 r}^{r+1}$ $=W_{2 g-2-2 r}^{g-r}$ by the Riemann-Roch Theorem, and since $2 \leq 2 g-2-2 r$ $\leq g-1$ this is the same as the first case.

Nöther's Theorem. If $W$ is not hyperelliptic, then every quadratic differential on $W$ can be written as the sum of (three) products of abelian differentials.

Communicated by J. Eells, Jr., May 13, 1968. Written with the support of the National Science Foundation. The material in this paper formed part of the author's thesis at Princeton University. The author is pleased to express his gratitude to his advisor, Professor Robert C. Gunning. 
Proof. The main step in the classical proof (see Hensel-Landsberg [3, p. 508]) is to find two abelian differentials $\omega_{1}$ and $\omega_{2}$ on $W$ which vanish simultaneously (counting multiplicities) at precisely $g-2$ points. By Theorem 1 , $\operatorname{dim} W_{g-1}^{2} \leq g-4$, so $\operatorname{dim} W_{g-1}^{2} \oplus-W_{1} \leq g-3<\operatorname{dim} W_{g-2}$. Hence it is possible to pick $g-2$ points $p_{1}, \cdots, p_{g-2}$ such that, for all $x \in W, p_{1}+\cdots$ $+p_{g-2}+x \notin W_{g-1}^{2}$, i.e. there is no non-constant meromorphic function on $W$ with poles at $p_{1}, \cdots, p_{g-2}$ and $x$ (counting multiplicities). Let $\omega_{1}$ and $\omega_{2}$ be two linearly independent abelian differentials vanishing at $p_{1}, \cdots, p_{g-2}$. Then by the Riemann-Roch Theorem $\omega_{1}$ and $\omega_{2}$ can have no other common zeroes, and moveover every abelian differential which vanishes at $p_{1}, \cdots, p_{g-2}$ must be a linear combination of $\omega_{1}$ and $\omega_{2}$.

Choose an abelian differential $\omega_{3}$ with no zeroes in common with either $\omega_{1}$ or $\omega_{2}$. Suppose that $\alpha_{1}, \alpha_{2}$. and $\alpha_{3}$ are three abelian differentials such that $\alpha_{1} \omega_{1}$ $+\alpha_{2} \omega_{2}+\alpha_{3} \omega_{3}=0$. It follows from the above remarks that $\alpha_{3}$ is a linear combination of $\omega_{1}$ and $\omega_{2}$, since it vanishes at $p_{1}, \cdots, p_{g-2}$. Suppose then that $\alpha_{1} \omega_{1}+\alpha_{2} \omega_{2}=0$. Then $\alpha_{2}$ vanishes at the $g$ points other than $p_{1}, \cdots, p_{g-2}$ where $\omega_{1}$ vanishes. But by the Riemann-Roch Theorem any abelian differential vanishing at these points must be a multiple of $\omega_{1}$. Therefore there are $3 g-3$ linearly independent quadratic differentials of the form $\alpha_{1} \omega_{1}+\alpha_{2} \omega_{2}+\alpha_{3} \omega_{3}$. But by the Riemann-Roch Theorem there are only $3 g-3$ linearly independent quadratic differentials in all; hence every quadratic differential can be written in the form $\alpha_{1} \omega_{1}+\alpha_{2} \omega_{2}+\alpha_{3} \omega_{3}$.

3. In this section we recall some of the basic properties of the subvarieties $W_{r}^{a}$, and calculate their dimension in the case where $W$ is hyperelliptic. All the results in this section occur in Mayer [4].

If $A$ and $B$ are subvarieties of a complex torus $J$, we define new subvarieties:

$$
\begin{aligned}
-A & =\{-a \mid a \in A\}, \\
A \oplus B & =\{a+b \mid a \in A, b \in B\}, \\
A \ominus B & =\{c \mid c+B \subseteq A\}=\cap\{A-b \mid b \in B\} .
\end{aligned}
$$

Here $-A$ is a subvariety since it is the inverse image of $A$ under multiplication by $-1 ; A \oplus B$ is a subvariety because it is the image of $A \times B$ under addition $\oplus: J \times J \rightarrow J$, which is a proper map (see the "Proper Mapping Theorem", Gunning and Rossi [2, p. 162]); finally, $A \ominus B$ is the intersection of the subvarieties $A-b$, and hence is itself a subvariety.

Theorem 2. $\operatorname{dim} W_{r}=r($ for $0 \leq r \leq g)$.

Proof. $W_{0}=\{0\}, W_{1}=W$, and $W_{g}=J(W)$ by Abel's Theorem, so Theorem 1 is true for $r=0,1, g$. In general $W_{r+1}=W_{r} \oplus W_{1}$, so $\operatorname{dim} W_{r+1}$ $\leq \operatorname{dim} W_{r}+1$. But since the extreme cases are known, we must have $\operatorname{dim} W_{r}=r$ for $0 \leq r \leq g$.

Lemma 1. $W_{r}^{a} \ominus-W_{1}=W_{r+1}^{a+1}($ for $r \geq 0, a \geq 1)$.

Proof. If a divisor class admits at least $a+1$ linearly independent mero- 
morphic functions, there will be at least a linearly independent combination of them which vanish at each point. Hence $W_{r+1}^{a+1} \oplus-W_{1} \subseteq W_{r}^{a}$, which implies $W_{r+1}^{a+1} \subseteq W_{r}^{a} \ominus-W_{1}$. On the other hand, if $d \in W_{r}^{a} \ominus-W_{1}$, then for every point $x \in W$ the divisor class $d-x$ admits at least a linearly independent meromorphic function. Hence among the meromorphic functions admitted by the divisor $d$, we can find, for each $x \in W$ which is not a point of $d$, at least a linearly independent one which vanishes at $x$. If the divisor $d$ did not admit at least $a+1$ linearly independent meromorphic functions, this would be impossible since then all the meromorphic functions admitted by $d$ would vanish everywhere, but $a \geq 1$. Therefore $W_{r}^{a} \ominus-W_{1} \subseteq W_{r+1}^{a+1}$.

Let $k$ be the divisor class of an abelian differential.

\section{Theorem 3.}

i) $k-W_{r}^{a}=W_{s}^{b}$, where $s=2 g-2-r$ and $b=g-1-r+a$.

ii) $W_{r-s}^{a} \ominus-W_{s}=W_{r}^{a+s}$ for $0 \leq s \leq r, a \geq 1$.

iii) $W_{r}^{a} \ominus W_{s}=W_{r-s}^{a}$ for $0 \leq s \leq r \leq g-1, a \geq 1$.

Proof. (i) is the Riemann-Roch Theorem. (ii) is true for $s=0$ (trivially) and for $s=1$ (by Lemma 1). Suppose it is true for $s=t \geq 1$. Then

$$
\begin{aligned}
& W_{r-t-1}^{a} \ominus-W_{t+1}=W_{r-t-1}^{a} \ominus\left[-W_{1} \oplus-W_{t}\right] \\
& \quad=\left[W_{r-t-1}^{a} \ominus-W_{1}\right] \ominus-W_{t}=W_{r-t}^{a+1} \ominus-W_{t}=W_{r}^{a+t+1} .
\end{aligned}
$$

Hence it is also true for $s=t+1$. Therefore by induction it is true for all $s$. To prove (iii), let $t=2 g-2-r$ and $b=g-1-r+a$. Then if $r \leq$ $g-1, b$ will be at least 1 and

$$
k-\left[W_{r}^{a} \ominus W_{s}\right]=\left[k-W_{r}^{a}\right] \ominus-W_{s}=W_{t}^{b} \ominus-W_{s}=W_{t+s}^{b+s}=k-W_{r-s}^{a} .
$$

Therefore $W_{r}^{a} \ominus W_{s}=W_{r-s}^{a}$.

Theorem 4. Let $1 \leq r \leq g-1$ and $a \geq 2$, and suppose $W_{r}^{a}$ is not empty. Then $\operatorname{dim} W_{r-1}^{a}+1 \leq \operatorname{dim} W_{r}^{a} \leq \operatorname{dim} W_{r-1}^{a-1}-1$.

Proof. Let $V$ be an irreducible subvariety of $W_{r-1}^{a}$ of maximal dimension; then $\operatorname{dim} V<g$. Since $W_{r-1}^{a} \oplus W_{1} \subseteq W_{r}^{a}, V \oplus W_{1} \subseteq W_{r}^{a}$. Moreover, $V \oplus W_{1}$ is irreducible, since it is the image of $V \times W_{1}$ under the addition map $\oplus: J(W)$ $\times J(W) \rightarrow J(W)$. If $\operatorname{dim} V \oplus W_{1}$ were equal to $\operatorname{dim} V$, we would have $V$ $=V \oplus W_{1}$, and by induction $V=V \oplus W_{g}$ which is impossible unless $V=\varnothing$ since $\operatorname{dim} V<g$. Therefore $\operatorname{dim} W_{r}^{a} \geq \operatorname{dim} W_{r-1}^{a}+1$, if $W_{r}^{a} \neq \varnothing$.

But we also have $W_{r}^{a} \oplus-W_{1} \subseteq W_{r-1}^{a-1}$. Let $X$ be an irreducible subvariety of $W_{r}^{a}$ of maximal dimension; then $0 \leq \operatorname{dim} X<g$. Since $X \oplus-W_{1} \subseteq W_{r-1}^{a-1}$ we conclude as before that $\operatorname{dim} X \oplus-W_{1}>\operatorname{dim} X$ and hence $\operatorname{dim} W_{r}^{a}$ $\leq \operatorname{dim} W_{r-1}^{a-1}-1$.

Theorem 5. Let $a \geq 1,1 \leq r \leq g-1$, and $r-2 a+2 \geq-1$. Then $\operatorname{dim} W_{r}^{a} \leq r-2 a+2$.

Proof. Applying the right hand side of the previous Theorem inductively, 
we deduce that

$$
\operatorname{dim} W_{r}^{a} \leq \operatorname{dim} W_{r-a+1}-(a-1)=r-2 a+2,
$$

provided that $W_{r}^{a}$ is not empty.

Theorem 6. If $W$ is hyperelliptic, and $r$ and $a$ are as above, then $\operatorname{dim} W_{r}^{a}$ $=r-2 a+2$.

Proof. Let $d$ be the divisor class of a meromorphic function $f$ on $W$ of order 2. Then the divisor class $n d$ admits $n+1$ linearly independent meromorphic functions $1, f, f^{2}, \cdots, f^{n}$. Therefore $\operatorname{dim} W_{2 n}^{n+1} \geq 0$. But applying the left hand side of Theorem 4 inductively we deduce that

$$
\operatorname{dim} W_{r}^{a} \geq \operatorname{dim} W_{2 a-2}^{a}+r-2 a+2 \geq r-2 a+2,
$$

provided that $r-2 a+2 \geq 0$.

4. We now observe that it is sufficient to prove Theorem 1 in the case $a=2$. For if $W_{r}^{a}$ is empty there is nothing to prove, and $W_{r}^{a}$ will always be empty if $a \geq 2$ and $r=1$. Otherwise we may apply the right hand side of Theorem 4 inductively to prove that $\operatorname{dim} W_{r}^{a} \leq \operatorname{dim} W_{r-a+2}^{2}-(a-2)$. Now if Theorem 1 is true for $a=2$, $\operatorname{dim} W_{r-a+2}^{2} \leq r-a-1$, which proves $\operatorname{dim} W_{r}^{a} \leq r-2 a+1$. Thus in general deficiencies in the dimensions of the $W_{r}^{2}$ will propagate themselves upward.

We may now reinterpret Theorem 1 by means of the following observation.

Theorem 6. $\operatorname{dim} W_{r}^{2}=r-2$ if and only if $W_{r-1}=W_{r}^{2} \oplus-W_{1}($ for $2 \leq r$ $\leq g-1)$.

Proof. Since $W_{r}^{2}=W_{r-1} \ominus-W_{1}$, we always have $W_{r}^{2} \ominus-W_{1} \subseteq W_{r-1}$. But $W_{r-1}$ is an irreducible subvariety, being the image under addition of a product of irreducible subvarieties (i.e. a product of $r-1$ copies of $W$ ). Therefore unless $W_{r}^{2} \oplus-W_{1}$ is equal to $W_{r-1}$, its dimension must be strictly smaller. On the other hand, the proof of Theorem 4 shows that $\operatorname{dim} W_{r}^{2}$ $<\operatorname{dim} W_{r}^{2} \oplus-W_{1}$. Therefore $\operatorname{dim} W_{r}^{2} \leq r-3$ unless $W_{r}^{2} \oplus-W_{1}=W_{r=1}$.

Hence, to complete the proof of Theorem 1, it is sufficient to prove the following theorem.

Theorem 7. If $W_{r}=W_{r+1}^{2} \oplus-W_{1}$ for some integer $r, 1 \leq r \leq g-2$, then $W$ is hyperelliptic.

5. To prove Theorem 7 , let $r$ be the smallest integer with $1 \leq r \leq g-2$ such that the hypothesis holds. If $r=1$, then $W_{2}^{2}$ is not empty and $W$ is hyperelliptic. We will show that if $r>1$ we get a contradiction.

Let $W_{(t)}$ be the Cartesian product of $W_{1}$ with itself $t$ times. An index of order $r$ is defined to be an unordered collection $A=\left\{i_{1}, \cdots, i_{r}\right\}$ of $r$ distinct elements of the set $\{1, \cdots, g-1\}$. Write $|A|=r$ and $p_{A}=p_{i_{1}}+\cdots+p_{i_{r}}$ for points $p_{1}, \cdots, p_{g-1}$.

Let $S$ be the subset of $W_{(g-1)}$ of all points $\left(p_{1}, \cdots, p_{g-1}\right)$ such that either

1) $p_{1}+\cdots+p_{g-1} \in W_{g-1}^{2}$, 
2) $p_{i}=p_{j}$ for some $i \neq j$,

3) $p_{A} \in W_{r}^{2}$ for some $A$ with $|A|=r$,

4) $p_{B} \in W_{r}^{2} \oplus-W_{1}$ for some $B$ with $|B|=r-1$, or

5) $p_{C}+p_{j} \in W_{r+1}^{2}$ for some $C$ with $|C|=r$.

Lemma 2. The set $S$ is a proper subvariety of $W_{(g-1)}$.

Proof. Conditions (1) and (3) define proper subvarieties since $\operatorname{dim} W_{r}^{2}$ $<\operatorname{dim} W_{r}$; condition (4) does since $W_{r-1} \not \subset W_{r}^{2} \oplus-W_{1}$ by the minimal choice of $r$. For condition (5), if $j \notin C$, this is the same as condition (3) for $A=C \cup j$; while if $j \in C$, write $C=j \cup D$. Suppose $2 p_{j}+p_{D} \in W_{r+1}^{2}$ for all $p \in W_{(g-1)}$. Then $2 W_{1} \oplus W_{r-1} \subseteq W_{r+1}^{2}$, or $2 W_{1} \subseteq W_{r+1}^{2} \ominus W_{r-1}=W_{2}^{2}$, so that $W_{2}^{2}$ is not empty and $W$ is hyperelliptic. Otherwise condition (5) must determine a proper subvariety. Since $W_{(g-1)}$ is irreducible, the union of a finite number of proper subvarieties will again be a proper subvariety.

Next let $Z$ be the subvariety of $W_{(g-1)} \times W_{1}$ of all points $\left(p_{1}, \cdots, p_{g-1}, q\right)$ such that for some indices $A \neq B$, both of length $r, p_{A}+q \in W_{r+1}^{2}$ and $p_{B}+q \in W_{r+1}^{2}$. Let $Q$ be the projection of $Z$ onto $W_{(g-1)} . Q$ is a subvariety by the "Proper Mapping Theorem" quoted above.

Lemma 3. $Q$ is a proper subvariety of $W_{(g-1)}$.

Proof. Let $A \neq B$ be two indices of length $r$. Let $k \in A$ but $k \notin B$. Write $A=k \cup L$ with $|L|=r-1, k \notin L$. Choose $p_{1}, \cdots, p_{k-1}, p_{k+1}, \cdots, p_{g-1}$ so that $p_{L} \notin W_{r}^{2} \oplus-W_{1}$ and $p_{B} \notin W_{r}^{2}$. There will be only finitely many $q$ with $p_{B}+q \in W_{r+1}^{2}$ (for otherwise $p_{B} \oplus W_{1} \subseteq W_{r+1}^{2}$ which implies $p_{B} \in W_{r+1}^{2} \ominus W_{1}$ $=W_{r}^{2}$ ). Then $p_{L}+q \notin W_{r}^{2}$ so by the same argument there are only finitely many choices of $q$ and $s$ with $p_{L}+q+s \in W_{r+1}^{2}$. Choose $p_{k}$ to be not one of these $s$. Then if $p_{B}+q \in W_{r+1}^{2}, p_{A}+q \notin W_{r+1}^{2}$. Thus the image of the subvariety $Z_{A B}=\left\{(p, q) \in W_{(g-1)} \times W_{1} \mid p_{A}+q \in W_{r-1}^{2}\right.$ and $\left.p_{B}+q \in W_{r-1}^{2}\right\}$ in $W_{(g-1)}$ does not contain $p$. Since $Q$ is the union of the images of the $Z_{A B}, Q$ is proper.

Lemma 4. If $p \in W_{r}$, there exists a $q \in W_{1}$ with $p+q \in W_{r+1}^{2}$.

Proof. $\quad W_{r} \subseteq W_{r+1}^{2} \oplus-W_{1}$. Thus we can write $p=r-q$ with $r \in W_{r+1}^{2}$ and $q \in W_{1}$, and $p+q=r$.

Now choose $p=\left(p_{1}, \cdots, p_{g-1}\right) \in W_{(g-1)}$ with $p \notin S$ and $p \notin Q$. For each $A$ with $|A|=r$ choose by Lemma 5 a point $q_{A} \in W_{1}$ with $p_{A}+q_{A} \in W_{r+1}^{2}$. Since $p_{A}+p_{j} \notin W_{\tau+1}^{2}$ for all $j, q_{A} \neq p_{j}$ for all $j$. Also if $|B|=r$ but $A \neq B$, then $q_{A} \neq q_{B}$, for otherwise we would have $\left(p, q_{A}\right) \in Z$ which cannot happen since $p \notin Q$. Since $p_{1}+\cdots+p_{g-1} \notin W_{g-1}^{2}$, there exists a non-zero abelian differential $\omega$ which vanishes at $p_{1}, \cdots, p_{g-1}$, and this condition determines $\omega$ uniquely up to a constant factor. But since $p_{A}+q_{A} \in W_{r+1}^{2}$, there exist $g-r$ linearly independent abelian differentials vanishing at $p_{i_{1}}, \cdots, p_{i_{r}}, q_{A}$ for each $A=\left\{i_{1}, \cdots, i_{r}\right\}$. Hence there is a non-zero abelian differential vanishing at $p_{1}, \cdots, p_{g-1}$ and at $q_{A}$, which is therefore a non-zero multiple of $\omega$. Hence $\omega$ vanishes at $q_{A}$ for each index $A$ of length $r$. Since $p_{1}, \cdots, p_{g-1}$ and the $q_{A}$ are 
all distinct, and there are $\left(\begin{array}{c}g-1 \\ r\end{array}\right)$ indices of length $r$, we get a contradiction if $(g-1)>g-1$. Hence either $r=1$ or $r=g-2$. If $r=1$ the surface is hyperelliptic as we saw before. Suppose $r=g-2$. If $g=3$ we are done also. Suppose $g \geq 4$.

Lemma 5. Let $X$ and $Y$ be analytic spaces and $V$ a proper subvariety of $X \times Y$. Then there exists a proper subvariety $S$ of $X$ such that if $x \notin S$ then $T(x)=\{y \in Y \mid(x, y) \in V\}$ is a proper subvariety of $Y$.

Proof. For each $y \in Y$, let $S(y)=\{x \in X \mid(x, y) \in V\}$. Then $S(y)$ is a subvariety of $X$, as is $S=\bigcap_{y \in Y} S(y)$. Since $V$ is proper, some pair $(x, y) \notin V$, and for this pair $x \notin S(y)$, so $x \notin S$. Hence $S$ is a proper subvariety of $X$. If $x \notin S$, then $x \notin S(y)$ for some $y$, and this pair $(x, y) \notin V$, so $y \notin T(x)$ $=\{y \in Y \mid(x, Y) \in V\}$ and $T(x)$ is a proper subvariety of $Y$.

Now regard $W_{(g-1)}=W_{(g-2)} \times W_{1}$, and let $L$ be a subvariety of $W_{(g-2)}$ such that if $\left(p_{1}, \cdots, p_{g-2}\right) \notin L$ then there exists a point $p_{g-1}$ with $\left(p_{1}, \cdots, p_{g-2}, p_{g-1}\right)$ $\notin S \cup Z$. This is possible by the previous lemma. Then the abelian differential $\omega$ constructed previously will vanish at the $2 g-2$ distinct points $p_{1}, \cdots, p_{g-1}$, $q_{1}, \cdots, q_{g-1}$, where we let $q_{i}=q_{A_{i}}$ with $A_{i}=\{1, \cdots, i-1, i+1, \cdots, g-1\}$ as the index obtained by omitting $i$. We chose $q_{g-1}$ so that $p_{1}+\cdots+p_{g-2}$ $+q_{g-1} \in W_{g-1}^{2}$, and we see now that if $p_{1}+\cdots+p_{g-2}+y \in W_{g-1}^{2}$, then there exist two linearly independent abelian differentials vanishing at $p_{1}, \cdots, p_{g-2}$ and $y$, and thus there is a non-zero abelian differential vanishing at $p_{1}, \cdots, p_{g-2}, y$ and $p_{g-1}$. But this must be a non-zero multiple of $\omega$. Thus $\omega$ will vanish at $y$. Since $\omega$ has precisely $2 g-2$ distinct zeroes, $y$ must be one of the points $p_{1}, \cdots, p_{g-1}, q_{1}, \cdots, q_{g-1}$. If $y=p_{j}$ we have $p_{A_{g-1}}+p_{j} \in W_{g-1}^{2}$ which contradicts condition (5) of the definition of $S$. If $y=q_{j}$ with $j \neq$ $g-1$, then $p_{A_{g-1}}+y \in W_{g-1}^{2}$ and $p_{A_{j}}+y \in W_{g-1}^{2}$ which contradicts the assumption that $\left(p_{1}, \cdots, p_{g-1}\right) \notin Z$. Hence $q_{g-1}$ is the unique point in $W_{1}$ with $p_{1}+\cdots+p_{g-2}+q_{g-1} \in W_{g-1}^{2}$; this proves

Lemma 6. There exists a proper subvariety $L$ of $W_{(g-2)}$ such that if $\left(p_{1}, \cdots, p_{g-2}\right) \notin L$ there is a unique point $q_{g-1}$ with $p_{1}+\cdots+p_{g-2}$ $+q_{g-1} \in W_{g-1}^{2}$.

Now fix a choice of $\left(p_{1}, \cdots, p_{g-3}\right)$ with $p_{1}+\cdots+p_{g-3} \notin W_{g-2}^{2} \oplus-W_{1}$ so that for at least one point $p_{g-2}$ we have $\left(p_{1}, \cdots, p_{g-3}, p_{g-2}\right) \notin L$. This is possible by Lemma 6. Let $R=\left\{\left(p_{g-2}, p_{g-1}\right) \in W_{1} \times W_{1} \mid p_{1}+\cdots+p_{g-1} \in W_{g-1}^{2}\right\}$. Then there are only finitely many $p_{g-2}$ for which $\left(p_{1}, \cdots, p_{g-2}\right) \in L$, and for all other choices of $p_{g-2}$ there is a unique $p_{g-1}$ with $\left(p_{g-2}, p_{g-1}\right) \in R$. Hence $R$ is a subvariety of dimension 1 , for it is proper and cannot be a finite set since it projects onto an infinite set in the first factor. Moreover, there, must be an irreducible component $N$ which projects onto the first factor, and this must be unique. In fact, $N$ is a 1-sheeted branched cover of $W_{1}$ under projection on the first factor. Consequently the projection of $N$ onto the first factor is one-to-one. 
Lemma 7. $N$ also projects onto the second factor.

Proof. If not, then $N$ projects onto a single point $p_{g-1}$. This means that $p_{1}+\cdots+p_{g-3}+p_{g-2}+p_{g-1} \in W_{g-1}^{2}$ for every $p_{g-2}$. Then $p_{1}+\cdots+p_{g-3}$ $+p_{g-1} \in W_{g-1}^{2} \ominus W_{1}=W_{g-2}^{2}$, and $p_{1}+\cdots+p_{g-3} \in W_{g-2}^{2} \oplus-W_{1}$, contrary to their choice.

Since the definition of $R$ is symmetric in $p_{g-2}$ and $p_{g-1}$, the projection of $N$ onto the second factor must also be one-to-one. Thus $N$ is the graph of a bianalytic automorphism of $W$ onto itself. Let $G$ be the group of all such maps. Since $g \geq 4$, the group $G$ is finite. Thus we have established

Lemma 8. There exists a proper subvariety $M$ of $W_{(g-3)}$ such that if $\left(p_{1}, \cdots, p_{g-3}\right) \notin M, p_{1}+\cdots+p_{g-3}+p_{g-2}+p_{g-1} \in W_{g-1}^{2}$, and $\left(p_{1}, \cdots, p_{g-2}\right)$ $\notin L$, then $p_{g-1} \in G p_{g-2}$ where $G p_{g-2}=\left\{g\left(p_{g-2}\right) \mid g \in G\right\}$.

Now choose $\left(p_{1}, \cdots, p_{g-2}\right)$ so that

1) $\left(p_{1}, p_{2}, \cdots, p_{g-2}\right) \notin L$,

2) $\left(p_{1}, p_{2}, \cdots, p_{g-4}, p_{g-2}, p_{g-3}\right) \notin L$,

3) $\left(p_{1}, p_{2}, \cdots, p_{g-3}\right) \notin M,\left(p_{1}, \cdots, p_{g-4}, p_{g-2}\right) \notin M$,

4) $p_{g-3} \notin G p_{g-2}$.

Then there exists a $p_{g-1}$ with $p_{1}+\cdots+p_{g-1} \in W_{g-1}^{2}$ since $W_{g-2}$ $\subset W_{g-1}^{2} \oplus-W_{1}$. Also by Lemma $8, p_{g-1} \in G p_{g-2}$ and $p_{g-1} \in G p_{g-3}$. Thus $p_{g-3}$ $\in G p_{g-2}$ which is a contradiction. This proves $r \neq g-2$ and completes the proof of Theorem 7 .

\section{References}

[1] R. C. Gunning, Lectures on Riemann surfaces, Princeton University Press, 1966.

[2] R. C. Gunning \& H. Rossi, Analytic functions of several complex variables, Prentice-Hall, Englewood Cliffs, N. J., 1965.

[3] K. Hensel \& G. Landsberg, Theorie der Algebraischen Funktionen einer Variabeln, Teubner, Leipzig, 1902.

[4] A. Mayer, On the Jacobi inversion theorem, Thesis, Princeton University.

CORNELL UNIVERSITY 
